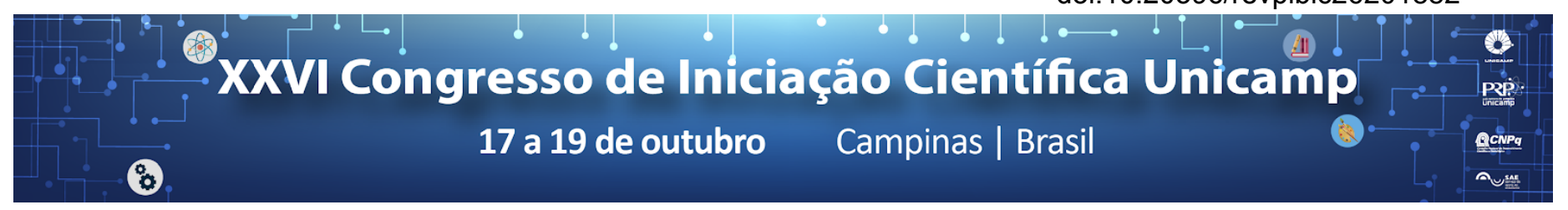

\title{
Modelos de teorias de gauge em mecânica clássica
}

\section{Aline Leite Vilela D’Oliveira*}

\section{Resumo}

Esse projeto teve como objetivo o estudo da teoria de fibrados principais e conexões. A partir de tal formalismo estudamos um exemplo de teoria de gauge em mecânica clássica, o movimento de um carro ao estacionar. Tal exemplo ilustra de maneira bastante completa a teoria de fibrados e conexões, o que foi útil para a compreensão mais profunda desses conceitos abstratos.

\section{Palavras-chave:}

Fibrados principais e conexões, teorias de gauge, fases geométricas.

\section{Introdução}

Do ponto de vista matemático, as teorias de gauge podem ser entendidas com o formalismo da teoria de fibrados e conexões. Nesse projeto, estudamos tal formalismo e, a partir do exemplo do movimento do carro, exemplificamos tal teoria de forma mais concreta.

\section{Resultados e Discussão}

Começamos com alguns conceitos teóricos [1].

Definição 1: Um fibrado principal $P(M, G, \pi)$ consiste das variedades diferenciáveis $P$ e $M$, do grupo de Lie $G$ e da projeção sobrejetora diferenciável $\pi: P \rightarrow M$, onde: 1 - $G$ age livremente e diferencialmente em $P$ à direita. 2 - M é o espaço quociente de $P$ pela relação de equivalência induzida por $\mathrm{G}$.

3 - P é localmente trivial: $\exists$ uma cobertura $\left\{U_{i}\right\}_{i \in I}$ de $M$, onde $\forall i \in I$ há um difeomorfismo $T_{i}: \pi^{-1}\left(U_{i}\right) \rightarrow U_{i} \times G$ (trivialização local). Ainda, $\forall p \in \pi^{-1}\left(U_{i}\right)$ temos $T_{i}(p)=\left(\pi(p), s_{i}(p)\right)$, com $s_{i}(p g)=s_{i}(p) g$.

Definição 2: Definimos o espaço vertical $V_{p} P$ em $p \in P$ como $V_{p} P=\left\{v \in T_{p} P /\left(\pi_{*}\right)_{p} v=0\right\}$.

Definição 3: Sejam $\mathfrak{g}$ a álgebra de Lie associada ao grupo de Lie $\mathrm{G}, A \in \mathfrak{g}, p \in P$ e $\gamma: \mathbb{R} \rightarrow G$ uma curva diferenciável qualquer tal que $\gamma(0)=e$ e $\left.\frac{d}{d t}(\gamma(t))\right|_{t=0}=A$. Definimos o vetor $A_{p}^{\#} \in V_{p} P$ como: $A_{p}^{\#}=\left.\frac{d}{d t}(p \cdot \gamma(t))\right|_{t=0}$.

Definição 4: Uma conexão associa a cada $p \in P$ um subespaço $H_{p} P$ de $T_{p} P$ tal que:

$1-T_{p} P=V_{p} P \oplus H_{p} P$.

2 - Dados $g \in G$ e $p \in P$ então $\left(\varphi_{g_{*}}\right)_{p} H_{p} P=H_{p g} P$.

3 - Dados $X$ um campo vetorial em $\mathrm{P}$ e $p \in \operatorname{dom}(X)$, existem $X^{H} \in H_{p} P$ e $X^{V} \in V_{p} P$ tais que $X_{p}=X^{H}+X^{V}$.

Equivalentemente podemos implementar o conceito de conexão através de uma 1-forma $\omega \in \Omega^{1}(p, \mathfrak{g})$, que permite achar as componentes vertical e horizontal de vetores em $T_{p} P$.

Definição 6: Seja $\gamma:[0,1] \rightarrow M$ uma curva suave. A curva $\bar{\gamma}:[0,1] \rightarrow P$ é um levantamento horizontal se:

$1-\pi \circ \bar{\gamma}=\gamma$.

2 - Qualquer vetor tangente a $\bar{\gamma}(t)$ pertence a $H_{\bar{\gamma}(t)} P$.

Teorema 2: Sejam $\gamma:[0,1] \rightarrow M$ uma curva suave em $M$ e $p_{0} \in \pi^{-1}(\gamma(0))$. Existe um único levantamento horizontal $\bar{\gamma}(t)$ em $\mathrm{P}$ tal que $\bar{\gamma}(0)=p_{0}$.
Fizemos o exemplo do estacionamento do carro representado na figura (1). O espaço de configurações total é $P$ e o espaço dos possíveis formatos do carro é $M$, com coordenadas $(\alpha, \beta, x, y, \varphi)$ e $(\alpha, \beta)$. Assim, temos a projeção $\pi: P \rightarrow M,(\alpha, \beta, x, y, \varphi) \mapsto(\alpha, \beta)$.
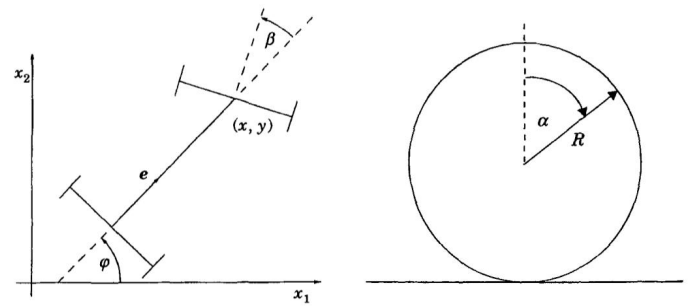

Figura 1. Coordenadas $(\alpha, \beta, x, y, \varphi)$. Retirada de [2].

Dado um elemento $\mathcal{B} \in E(2)$ podemos definir a ação $\varphi_{\mathcal{B}}: P \rightarrow P$ como o movimento rígido $\mathcal{B}$ do vetor $\widehat{e}$ da figura (1). Assim, $P(M, E(2), \pi)$ é um fibrado principal.

Impondo que a roda não deslize na pista, temos uma conexão onde os vetores que geram o espaço horizontal, $H_{\alpha}$ e $H_{\beta}$, dã as direções permitidas para o movimento do carro em $P$. Seja $\gamma(t)=(\alpha(t), \beta(t))$ uma curva em . Seu levantamento horizontal é a curva dada por $\frac{d}{d t} \bar{\gamma}(t)=\frac{d}{d t} \alpha(t) H_{\alpha}+\frac{d}{d t} \beta(t) H_{\beta}$ com condição inicial $\left(x_{0}, y_{0}, \varphi_{0}\right)$.

Nosso objetivo é estacionar o carro, ou seja, movê-lo lateralmente. Assim, realizamos um ciclo infinitesimal em $P$ gerado pelos vetores $H_{\alpha}$ e $\left[H_{\alpha}, H_{\beta}\right]$. Seja $\varphi_{t}^{\left[H_{\alpha},\left[H_{\alpha}, H_{\beta}\right]\right]}$ o fluxo do campo vetorial $\left[H_{\alpha},\left[H_{\alpha}, H_{\beta}\right]\right]$ em $P$, e $\left(\alpha_{0}, \beta_{0}, x_{0}, y_{0}, \varphi_{0}\right) \in P$ a posição inicial do carro. Então, a posição final do carro será: $\varphi_{\varepsilon^{4}}^{\left[H_{\alpha},\left[H_{\alpha}, H_{\beta}\right]\right]}\left(\alpha_{0}, \beta_{0}, x_{0}, y_{0}, \varphi_{0}\right)$ $=\left(\alpha_{0}, \beta_{0}, x_{0}+\varepsilon^{4} \frac{R^{2}}{l} \sin \varphi_{0}, y_{0}-\varepsilon^{4} \frac{R^{2}}{l} \cos \varphi_{0}, \varphi_{0}\right)$.

Logo, conseguimos estacionar o carro a partir de um ciclo de movimentos permitidos em $P$.

\section{Conclusões}

Utilizando os conceitos de fibrados principais e conexões fizemos o exemplo do estacionamento do carro, onde a conexão carrega a informação do não deslizamento da roda do carro na pista.

Esse formalismo pode ser aplicado a outros sistemas físicos. Como, por exemplo, o efeito swimming de corpos articulados em espaços curvos.

${ }^{1}$ Kobayashi S, Nomizu K. Foundations of differential geometry. Vol 1. New York: Interscience publishers; 1963.

${ }^{2}$ Fecko M. Gauge-potential approach to the kinematics of a moving car. Il Nuovo Cimento 1996; 111B(11): 1315-1332. 